\title{
Necromassa em reflorestamentos com espécies nativas da Mata Atlântica com 4, 6 e 8 anos de implantação
}

\author{
Mariana Chaves Mota ${ }^{1}$ e José Marcelo Domingues Torezan ${ }^{1,2}$
}

Recebido: 17.06.2013; aceito: 04.09.2013

\begin{abstract}
Necromass in 4, 6 and 8-year old Atlantic Forest restoration sites). In addition to the recognized contribution of ecological restoration to ecosystem services, the fixation of atmospheric carbon has gained attention, as a result of the carbon uptake by growing trees, which can accumulate in different compartments, including the necromass. The goal of this study was to identify factors that explain the variability in necromass amount in reforestations with native species after a period of 4,6 , and 8 years of implantation. Necromass amounts were higher with higher age of implantation, canopy cover, and abundance of woody species, being the medium and large branches the components most influenced by the factors analyzed. Necromass was also associated with increase in concentration of organic carbon in the soil, suggesting that, in older reforestations, with denser canopies, microclimatic conditions can favor not only the deposition but also decomposition of necromass.
\end{abstract}

Key words: dead plant biomass, forest restoration, litter, soil organic carbon

RESUMO - (Necromassa em reflorestamentos com espécies nativas da Mata Atlântica com 4, 6 e 8 anos de implantação). Além dos serviços ambientais reconhecidos da restauração ecológica, tem sido ressaltada a função de fixação de carbono atmosférico, que pode se acumular em diferentes compartimentos, inclusive na necromassa. Este estudo objetivou identificar fatores que expliquem a variação da necromassa em reflorestamentos com espécies nativas com diferentes idades de implantação (4, 6 e 8 anos). Os resultados indicam que a necromassa aumentou com a idade, a cobertura do dossel e a abundância de espécies lenhosas, sendo os galhos médios e grandes os componentes mais influenciados pelos fatores analisados. Quanto maior a deposição de necromassa maior a concentração de carbono orgânico no solo, o que sugere que reflorestamentos mais antigos e, consequentemente, com dossel mais denso, possibilitam condições microclimáticas mais favoráveis tanto para o processo de deposição quanto para o de decomposição da necromassa.

Palavras-chave: biomassa vegetal morta, carbono orgânico do solo, restauração ecológica, serapilheira

\section{Introdução}

Dentre as principais metas associadas à restauração ecológica inclui-se a proteção dos recursos abióticos, como solo e água, e o restabelecimento da diversidade biológica e dos processos ecológicos do ecossistema degradado (Engel \& Parrota 2008, Hobbs 2008, Martins 2012). Mais recentemente, a fixação de carbono atmosférico pelas árvores em crescimento tem ganhado importância em decorrência de sua possível influência nas mudanças climáticas, ressaltando esse serviço ambiental prestado pelos reflorestamentos (Pawson et al. 2013).

Uma parte do carbono fixado na fotossíntese é utilizada na formação dos tecidos da planta, sendo na madeira do caule acumulada a maior porção e de maneira mais duradoura (Nutto et al. 2002, Raven et al. 2007). Contudo, o acúmulo de carbono também ocorre nas demais partes da planta, que compreendem as folhas, flores, frutos e sementes. Esses fragmentos orgânicos senescentes, ao caírem sobre o solo, formam a serapilheira (White et al. 2012, Caldeira et al. 2013).

A serapilheira juntamente com as árvores tombadas, as mortas em pé, os galhos e os troncos caídos sobre o solo formam a biomassa vegetal morta, ou necromassa, do ecossistema florestal (Palace et al. 2012). A deposição da necromassa é indicadora de produtividade primária e pode ser influenciada por diversos fatores, tais como: temperatura, umidade,

1. Universidade Estadual de Londrina, Centro de Ciências Biológicas, Departamento de Biologia Animal e Vegetal, Laboratório de Biodiversidade e Restauração de Ecossistemas, Rodovia Celso Garcia Cid, Caixa Postal 6001, 86051-970 Londrina, PR, Brasil

2. Autor para correspondência: torezan@uel.br 
relevo, tipo de vegetação, precipitação, deciduidade, estádio sucessional, disponibilidade hídrica, características do solo e regimes de luminosidade, dentre outros (Figueiredo Filho et al. 2003, Palace et al. 2012, Kuruppuarachchi \& Seneviratne 2013, Lv et al. 2013).

A necromassa proporciona uma fonte constante de matéria orgânica para a ciclagem de carbono e de nutrientes (Palace et al. 2012, Bellingham et al. 2013). Sua camada depende, além da produção e deposição, da velocidade de decomposição da matéria orgânica, que varia conforme a composição do substrato, atividade dos decompositores e das condições ambientais, particularmente temperatura e umidade (Mielniczuk et al. 2003, Bellingham et al. 2013).

O conhecimento dos processos de produção, deposição e acúmulo de necromassa é importante para compreender a dinâmica do carbono em florestas tropicais, uma vez que o vegetal ao morrer deixa de acumular carbono e passa a liberá-lo para o solo e para a atmosfera (Palace et al. 2008, Palace et al. 2012). Embora contribua com uma porção considerável da biomassa total acima do solo (Palace et al. 2007, Kuruppuarachchi \& Seneviratne 2013), a necromassa ainda é pouco estudada em florestas tropicais e, em menor frequência, em ecossistemas florestais em restauração.

Desse modo, este estudo objetivou identificar fatores ambientais que influenciam a deposição de necromassa em reflorestamentos com espécies nativas, bem como avaliar se a idade do reflorestamento é um dos fatores que contribuem para essa deposição.

\section{Material e métodos}

Área de estudo - Os reflorestamentos com espécies nativas selecionados para este estudo estão situados no entorno do Reservatório de Capivara, na bacia do rio Paranapanema, no norte do Estado do Paraná, Brasil. A região era originalmente recoberta por Floresta Estacional Semidecidual (IBGE 2004). O índice pluviométrico regional médio é de $1.400 \mathrm{~mm}^{-1}$ e a temperatura média é superior a $22{ }^{\circ} \mathrm{C}$ nos meses mais quentes e inferior a $18{ }^{\circ} \mathrm{C}$ nos meses mais frios (IAPAR 2000).

Os três sítios selecionados apresentam uniformidade de altitude (334-338 m s.n.m.), relevo (suave), clima (Cfa de Köppen), tipologia de solo (Nitossolo vermelho eutrófico e Latossolo vermelho eutroférrico) (EMBRAPA 2011), histórico de ocupação (sojicultura desde os anos 1970), matriz agrícola circundante (sojicultura) e técnicas de implantação (ver a seguir), restando como variável apenas a data de implantação.

Os reflorestamentos foram implantados com preparo mecanizado do solo, com mudas produzidas em tubetes de $50 \mathrm{ml}$, sem adubação ou calagem, em espaçamento $3 \times 2 \mathrm{~m}$. A manutenção resumiu-se a capina manual ou mecânica e controle de formigas cortadeiras, por dois anos. A mistura de espécies empregada privilegiou as de rápido crescimento, tais como: Heliocarpus americanus L. e Guazuma ulmifolia Lam. (Malvaceae), e Trema micrantha (L.) Blume (Cannabaceae), que compreenderam cerca de $50 \%$ das mudas.

Coleta de dados - Foram selecionados três reflorestamentos com diferentes idades de implantação (quatro, seis e oito anos). Em cada reflorestamento foram dispostas 10 parcelas de $10 \times 10 \mathrm{~m}\left(100 \mathrm{~m}^{2}\right)$ cada, respeitando a distância mínima de $20 \mathrm{~m}$ entre as parcelas e equidistância das bordas do reflorestamento. A biomassa vegetal morta (necromassa) foi coletada, por parcela, durante o período de verão e dividida em três componentes:

Árvores mortas em pé ou tombadas sobre o solo A biomassa dos troncos foi estimada por meio da multiplicação do volume pela densidade da madeira. Para estimar o volume do tronco foi calculado o volume de um cilindro cujo diâmetro corresponde à média de dois valores de diâmetro mensurados. A altura foi estimada visualmente com o auxílio de um bastão graduado. Para obter a densidade da madeira foram coletados discos amostrais (casca + alburno + cerne) de todos os indivíduos encontrados na parcela. Os discos amostrais tiveram o volume mensurado por imersão em água em um recipiente graduado, e foram secos a $80^{\circ} \mathrm{C}$ até massa constante. A densidade da madeira dos discos foi obtida dividindo-se a massa pelo volume (Vital 1984, Trugilho et al.1990) e utilizada no cálculo da biomassa dos troncos.

Galhos sobre o solo - Para galhos grandes ( $\geq 5 \mathrm{~cm} \varnothing)$ os procedimentos empregados foram os mesmos aplicados às árvores mortas. Para galhos médios $(\geq 1 \mathrm{~cm} \varnothing \mathrm{e}<5 \mathrm{~cm} \varnothing)$ foi estabelecida uma subparcela de $5 \times 5 \mathrm{~m}\left(25 \mathrm{~m}^{2}\right)$ onde todos os galhos foram recolhidos e pesados em campo para determinação da massa fresca. Desse material, foi retirada uma alíquota pesada em campo para determinação do conteúdo de água (por meio de secagem em estufa a $80{ }^{\circ} \mathrm{C}$ ) e posterior transformação dos valores de massa 
fresca em massa seca. Os galhos pequenos $(<1 \mathrm{~cm} \mathrm{Ø)}$ foram incorporados à coleta de serapilheira (Palace et al. 2008).

Serapilheira - A serapilheira foi coletada no centro de cada parcela, com o auxílio de um gabarito quadrado de $1 \times \mathrm{m}\left(1 \mathrm{~m}^{2}\right)$, e seca em estufa a $80{ }^{\circ} \mathrm{C}$ até massa constante.

Adicionalmente, a cobertura do dossel foi estimada por meio de fotografias hemisféricas com lente "olho de peixe" (distância focal de $8 \mathrm{~mm}$ ), tomadas a $1 \mathrm{~m}$ do solo e analisadas com auxílio do Software Gap Light Analyzer vs. 2.0 (Frazer et al. 1999). A abundância de espécies lenhosas foi determinada por meio da contagem de todos os indivíduos lenhosos (arbustivos ou arbóreos) com $\mathrm{DAB} \geq 5 \mathrm{~cm}$ encontrados na parcela de $10 \times 10 \mathrm{~m}$. Para a determinação do carbono orgânico do solo foi coletada uma amostra de aproximadamente $500 \mathrm{~g}$ de solo por parcela, obtida a partir de quatro perfurações de zero a $20 \mathrm{~cm}$ de profundidade. As amostras foram enviadas para análise no Laboratório de Solos do IAPAR, em Londrina-PR.

Análise de dados - A relação existente entre as variáveis com distribuição normal e que apresentaram homogeneidade de variâncias, segundo o teste de Shapiro-Wilk e Levene, respectivamente, foi avaliada por meio da análise de regressão e da análise de variância (ANOVA), seguida do teste de Tukey. Para as variáveis que apresentaram distribuição significativamente diferente da normal e/ ou heterogeneidade de variâncias foram empregados o coeficiente de correlação de Spearman e o teste de Kruskal-Wallis, seguido do teste de Mann-Whitney. Para todos os testes foi considerado $\alpha=0,05$.

\section{Resultados}

A necromassa do reflorestamento de quatro anos estava distribuída em $5.210 \mathrm{~g} \mathrm{~m}^{-2}$ de serapilheira, $346 \mathrm{~g} \mathrm{~m}^{-2}$ de galhos médios, $43 \mathrm{~g} \mathrm{~m}^{-2}$ de galhos grandes e $21 \mathrm{~g} \mathrm{~m}^{-2}$ de árvores mortas, totalizando $5.622 \mathrm{~g} \mathrm{~m}^{-2}$ No reflorestamento de seis anos foram encontrados $5.550 \mathrm{~g} \mathrm{~m}^{-2}$ de serapilheira, $226 \mathrm{~g} \mathrm{~m}^{-2}$ de galhos médios, $125 \mathrm{~g} \mathrm{~m}^{-2}$ de galhos grandes e $2.168 \mathrm{~g} \mathrm{~m}^{-2}$ de árvores mortas em pé ou tombadas, totalizando $9.392 \mathrm{~g} \mathrm{~m}^{-2}$. O reflorestamento de oito anos apresentou $4.639 \mathrm{~g} \mathrm{~m}^{-2}$ de serapilheira, $1.326 \mathrm{~g} \mathrm{~m}^{-2}$ de galhos médios, $631 \mathrm{~g} \mathrm{~m}^{-2}$ de galhos grandes e $4.181 \mathrm{~g} \mathrm{~m}^{-2} \mathrm{de}$ árvores mortas, totalizando $10.779 \mathrm{~g} \mathrm{~m}^{-2}$ (figura 1).

Segundo a análise de variância (ANOVA), a deposição de necromassa total (serapilheira + galhos médios e grandes + árvores mortas) apresentou diferença significativa entre os reflorestamentos com diferentes idades de implantação $\left(\mathrm{F}_{2,27}=3,24 ; p<0,05\right.$; figura 2a), sendo o de quatro e o de oito anos diferentes entre si (teste Tukey; $p<0,05$ ), com maior necromassa encontrada no de oito anos; o de seis anos não diferiu dos demais reflorestamentos (teste Tukey; $p>0,05$ ). As deposições de galhos médios (ANOVA; $F_{2,27}=16,46$; $\mathrm{p}<0,001$; figura $2 \mathrm{~b}$ ) e grandes (Kruskal-Wallis; $H=10,91 ; p<0,01)$ também apresentaram diferença entre os reflorestamentos, tendo o de oito anos maior necromassa quando comparado ao de quatro e ao de seis anos, tanto para a deposição de galhos médios (teste Tukey; $\mathrm{p}<0,001$ ), quanto para a de galhos grandes (Mann-Whitney; $\mathrm{p}<0,01$ ).

Não houve diferença significativa entre os reflorestamentos com diferentes idades para a deposição de serapilheira (ANOVA; $\mathrm{F}_{2,27}=16,46$; $\mathrm{p}>0,05)$ e de árvores mortas em pé ou tombadas sobre o solo (Kruskal-Wallis; $\mathrm{H}=3,42 ; \mathrm{p}>0,05$ ).

A cobertura do dossel do reflorestamento apresentou correlação significativa e positiva tanto com a deposição de necromassa total (regressão; $\mathrm{r}^{2}=0,16 ; \mathrm{p}<0,05$, figura 3a) quanto de galhos médios (regressão; $\mathrm{r}^{2}=0,47 ; \mathrm{p}<0,001$, figura $3 \mathrm{~b}$ ) e grandes (Spearman; $r_{s}=0,42 ; p<0,01$; tabela 1). Já a deposição de serapilheira (regressão; $\mathrm{r}^{2}=0,04$; p > 0,05) e de árvores mortas em pé ou tombadas sobre o solo (Spearman; $r_{s}=0,24 ; p>0,05$ ) não se correlacionaram significativamente com a cobertura do dossel.

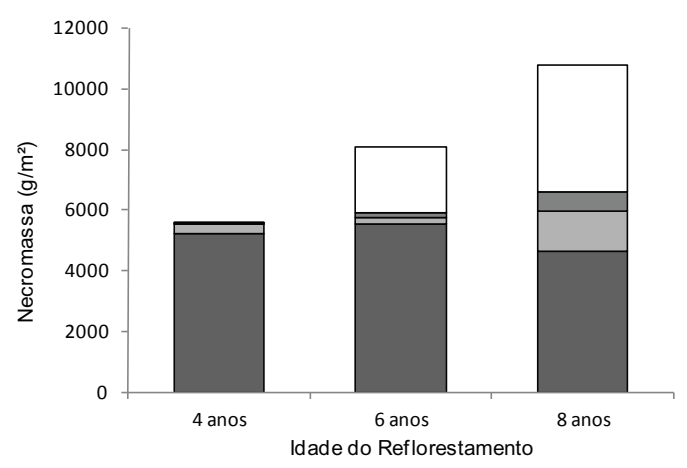

Figura 1. Distribuição dos componentes da necromassa, em g.m ${ }^{-2}$, nos reflorestamentos com diferentes idades (anos) localizados no Norte do Estado do Paraná, Brasil. $\square$ Árvores mortas, $\square$ Galhos grandes, $\square$ Galhos médios, $\square$ Serrapilheira.

Figure 1. Distribution of necromass components, in $\mathrm{g} . \mathrm{m}^{-2}$, in reforestations with different ages (years) located in Northern Paraná State, Brazil. $\square$ Árvores mortas, Galhos grandes, - Galhos médios, - Serrapilheira. 
De acordo com o teste de Correlação de Spearman, a deposição de galhos médios $\left(\mathrm{r}_{\mathrm{s}}=0,60 ; \mathrm{p}<0,001\right)$ e de galhos grandes $\left(r_{s}=0,59 ; p<0,001\right)$ apresentaram correlação significativa e positiva com a abundância de espécies lenhosas (tabela 1), o que não ocorreu para a deposição de necromassa total $\left(r_{s}=0,24 ; p>0,05\right)$, de serapilheira $\left(r_{s}=-0,06 ; p>0,05\right)$ e de árvores mortas em pé ou tombadas sobre o solo $\left(r_{s}=0,11 ; p>0,05\right)$, onde não houve correlação significativa.

De acordo com a análise de variância (ANOVA), houve diferença significativa entre os reflorestamentos com diferentes idades em relação à cobertura do dossel $\left(\mathrm{F}_{2,27}=36,67 ; \mathrm{p}<0,001\right)$, tendo o reflorestamento de oito anos dossel mais fechado quando comparado ao de quatro e ao de seis anos (teste Tukey; $\mathrm{p}<0,001$ ). Assim como a abundância de espécies lenhosas (Kruskal-Wallis; $\mathrm{H}=13,38 ; \mathrm{p}<0,001$ ), que apresentou maior valor no reflorestamento de oito anos, quando comparado ao de quatro e ao de seis anos (MannWhitney; $\mathrm{p}<0,01)$. A cobertura do dossel apresentou correlação significativa e positiva com a abundância de espécies lenhosas (Spearman; $r_{s}=0,43 ; p<0,01$ ). Segundo a análise de regressão, quanto maior a deposição de necromassa total maior a concentração de carbono orgânico no solo do reflorestamento $\left(\mathrm{r}^{2}=0,15 ; \mathrm{p}<0,05\right)$.

\section{Discussão}

Os resultados apontam que a idade de implantação, a abundância de espécies lenhosas e a cobertura do dossel influenciam a deposição de necromassa em reflorestamentos com espécies nativas, sendo os galhos médios e grandes os componentes da necromassa que mais responderam às variações dos fatores analisados. As deposições de serapilheira e de árvores mortas podem estar respondendo a outros fatores não analisados neste estudo, como sazonalidade (Durigan et al. 1996) e estresse hídrico (Nepstad et al. 2007).

Quanto mais antigo o reflorestamento maior a abundância de espécies lenhosas, que ocasiona uma maior complexidade estrutural e, consequentemente, uma cobertura do dossel mais densa. Melo \& Durigan (2007) e Melo et al. (2007) também observaram evoluções estruturais ao longo do tempo em reflorestamentos com espécies nativas localizados no Médio Vale do Paranapanema-SP, onde reflorestamentos mais antigos apresentaram maiores porcentagens de cobertura do dossel. No entanto, são necessárias amostragens em uma faixa de idades mais ampla para caracterizar melhor os padrões de mudança na deposição de necromassa com o tempo, especialmente em plantios muito jovens (i.e., menos de cinco anos) e mais antigos que os incluídos neste estudo.

A cobertura do dossel apresenta relação direta com a quantidade, qualidade e distribuição temporal e espacial da luz, o que determina níveis diferenciados de temperatura e condições de umidade do ar e do solo (Jennings et al.1999, Maciel et al. 2002, Rebetez et al. 2012). Segundo Campbell \& Norman (1989), o tipo de estrutura do dossel pode ocasionar ainda consequências indiretas, como variações nos processos de fotossíntese, transpiração, infecção por
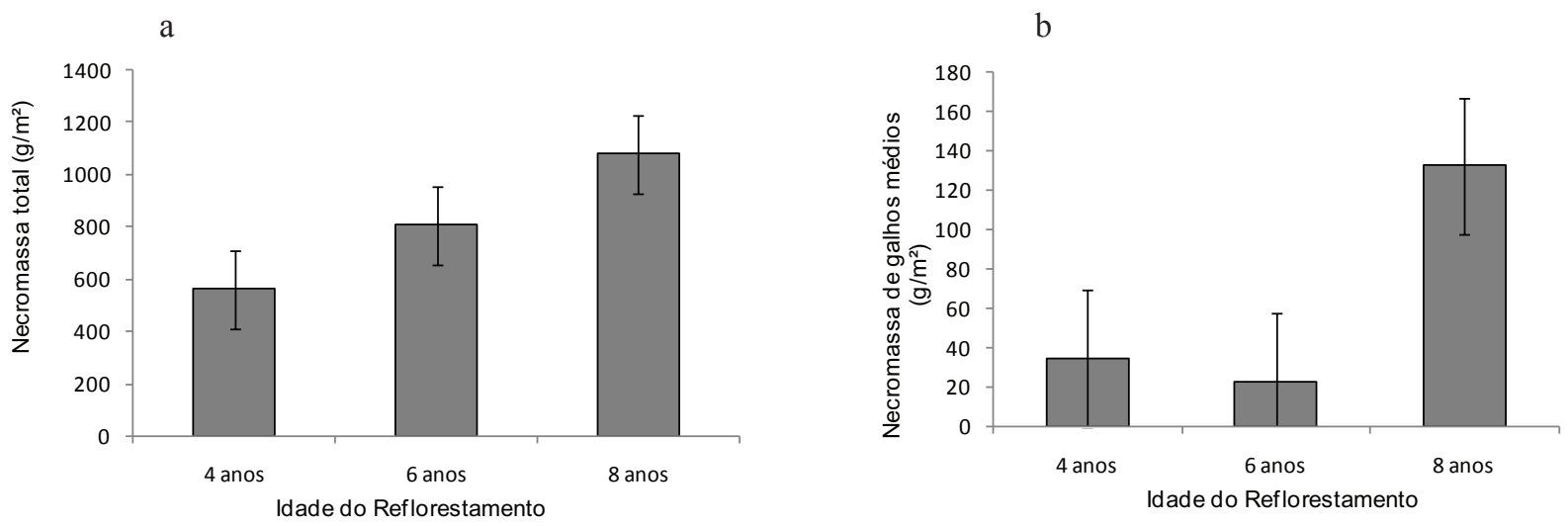

Figura 2. Deposição da necromassa total (a) e dos galhos médios (b) em reflorestamentos com diferentes idades de implantação (anos) localizados no Norte do Paraná, Brasil. As barras indicam o erro padrão.

Figure 2. Deposition of total necromass (a) and necromass of medium branches (b) in reforestations with different ages of implantation (years) located in Northern Paraná State, Brazil. The bars indicate the standard error. 

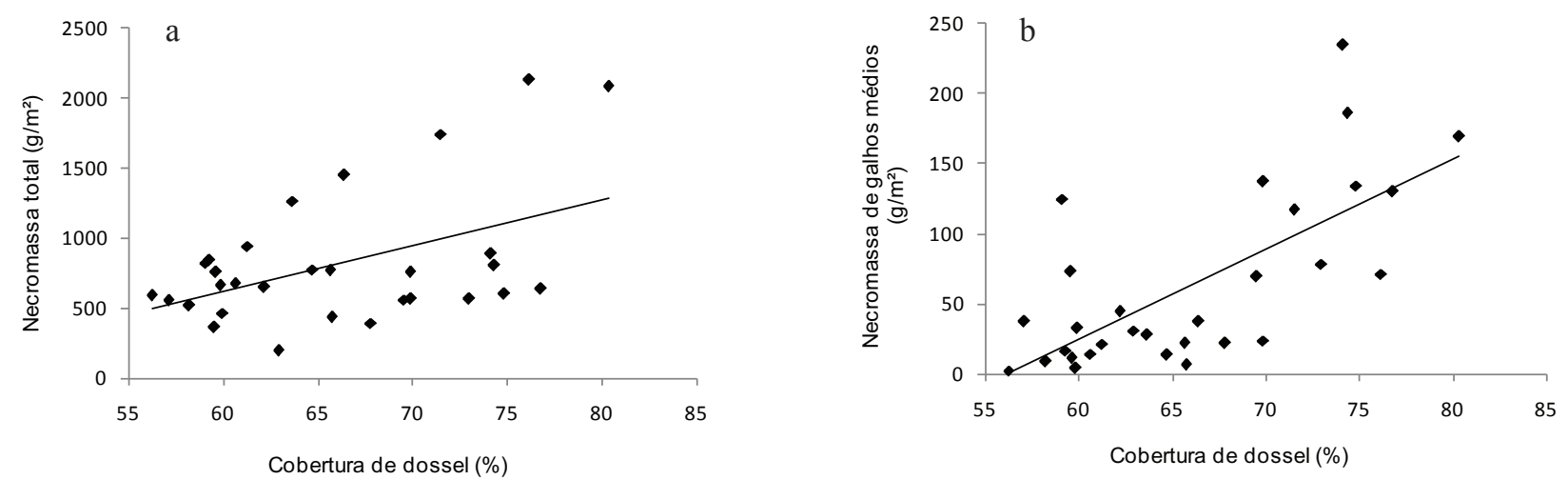

Figura 3. Análise de regressão linear entre a cobertura do dossel e a necromassa total (a) e a necromassa de galhos médios (b) depositadas em reflorestamentos com espécies nativas localizados no Norte do Paraná, Brasil.

Figure 3. Linear regression analysis between canopy cover and total necromass (a) and necromass of medium branches (b) deposited in reforestations with native species located in Northern Paraná State, Brazil.

patógenos, multiplicação de insetos e competição na comunidade de plantas.

Esses fatores, isolados ou em conjunto, podem influenciar a deposição dos galhos situados nas camadas inferiores às copas das árvores, tendo em vista que a queda de galhos, como um processo fisiológico complexo, é influenciado por diversos fatores externos e internos como luminosidade, umidade, temperatura, ação mecânica do vento e da chuva, fator genético, insetos, doenças e idade do indivíduo lenhoso (Kozlowski \& Pallardy 1996, Martins \& Rodrigues 1999, Portela \& Santos 2007, Bellingham et al. 2013, Lv et al. 2013).

A maior deposição de necromassa em reflorestamentos mais antigos e estruturalmente mais complexos influencia diretamente a concentração de carbono orgânico no solo, atribuindo aos reflorestamentos um papel importante na mitigação das mudanças climáticas (Melo \& Durigan 2006, Pawson et al. 2013). Em ecossistemas terrestres, o compartimento de carbono do solo pode exceder em até quatro vezes o da parte aérea da vegetação, evidenciando a extrema importância do solo na dinâmica global do carbono (Machado 2005, IPCC 2007, Nave et al. 2010). Outros estudos também têm constatado que a simples deposição de necromassa pode explicar o aumento na concentração de carbono orgânico no solo, como Rangel et al. (2007), que ao depositar resíduos vegetais em entrelinhas de cafeeiro verificaram aumento no teor de carbono orgânico no solo em comparação às áreas onde não houve deposição.

A necromassa depositada permanece sobre o solo até ser fragmentada e decomposta pelos processos físicos, químicos e bióticos, que são influenciados, principalmente, pela temperatura e umidade do ambiente (Mielniczuk et al. 2003, Bellingham et al. 2013). Ainda que o processo de decomposição da necromassa libere uma parte do carbono para a atmosfera em forma de $\mathrm{CO}_{2}$, o restante do carbono decomposto passa a fazer parte da matéria orgânica (húmus), como um componente do solo (Santos \& Camargo 1999). Como a cobertura do dossel influencia indiretamente as condições de temperatura e umidade do ambiente (Jennings et al.1999, Maciel et al. 2002,

Tabela 1. Coeficientes de Correlação de Spearman $\left(r_{s}\right)$ entre a cobertura do dossel e a abundância de espécies lenhosas e os componentes da necromassa depositados em reflorestamentos com espécies nativas localizados no Norte do Estado do Paraná, Brasil. * Correlação significativa; $p<0,01$.

Table 1. Spearman correlation coefficients $\left(r_{s}\right)$ between canopy cover and abundance of alive woody species alive and the necromass components deposited in reforestations with native species located in northern Paraná State, Brazil. * Significant correlation; $p<0,01$.

\begin{tabular}{|c|c|c|c|c|c|}
\hline & $\begin{array}{c}\text { Necromassa total } \\
r_{s}\end{array}$ & $\begin{array}{c}\text { Serapilheira } \\
\mathrm{r}_{\mathrm{s}}\end{array}$ & $\begin{array}{c}\text { Galhos médios } \\
\mathrm{r}_{\mathrm{s}}\end{array}$ & $\begin{array}{c}\text { Galhos grandes } \\
\mathrm{r}_{\mathrm{s}}\end{array}$ & $\begin{array}{c}\text { Árvores mortas } \\
r_{\mathrm{s}}\end{array}$ \\
\hline Cobertura do dossel (\%) & & & & $0,42 *$ & 0,24 \\
\hline Abundância de lenhosas (ind.ha-1) & 0,24 & $-0,06$ & $0,60 *$ & $0,59 *$ & 0,11 \\
\hline
\end{tabular}


Rebetez et al. 2012), reflorestamentos estruturalmente mais complexos possibilitam um microclima mais favorável que pode estar contribuindo tanto para o processo de deposição quanto para o de decomposição da necromassa, ocasionando uma maior concentração e estoque de carbono orgânico no solo. No entanto, são necessários estudos que incluam o monitoramento permanente de variáveis microclimáticas, especialmente umidade do ar e do solo, concomitantemente ao registro da deposição da necromassa.

Portanto, conclui-se que a deposição de necromassa nos reflorestamentos amostrados está sendo influenciada pela idade de implantação e, consequentemente, pela abundância de espécies lenhosas e pela cobertura do dossel. Quanto maior a deposição de necromassa maior a concentração de carbono orgânico no solo, que também pode estar sendo influenciada pela cobertura do dossel mais densa de áreas mais antigas e estruturalmente mais complexas, que podem possibilitar condições microclimáticas mais favoráveis tanto para o processo de deposição quanto para o de decomposição da necromassa.

\section{Literatura citada}

Bellingham, P.J., Morse, C.W., Buxton, R.P., Bonner, K.I., Mason, N.W. \& Wardle, D.A. 2013. Litterfall, nutrient concentrations and decomposability of litter in a New Zealand temperate montane rain forest. New Zealand Journal of Ecology 37: 162-171.

Caldeira, M.V.W., da Silva, R.D., Kunz, S.H., Zorzanelli, J.P.F., Castro, K.C. \& de Oliveira, T.G. 2013. Biomassa e nutrientes da serapilheira em diferentes coberturas florestais, Alegre-ES. Comunicata Scientiae 4: 111-119.

Campbell, C.S. \& Norman, J.M. 1989. The description and measurement of plant canopy structure. In: G. Russell, B. Marshall \& P.G Jarvis (orgs.). Plant canopies: Their growth, form and function. Cambridge University, Cambridge.

Durigan, G., Leitão Filho, H.F. \& Pagano, S.N. 1996. Produção de folhedo em matas ciliares na região oeste do Estado de São Paulo. Revista do Instituto Florestal 8: 187-199.

EMBRAPA - Empresa Brasileira de Pesquisa Agropecuária. 2011. Mapa de solos do Brasil. http:// www.cnps.embrapa.br/ (acesso em 31.01.2013).

Engel, V.L. \& Parrotta, J.A. 2008. Definindo a restauração ecológica: tendências e perspectivas mundiais. 1ed. revisada. In: P.Y. Kageyama, R.E. Oliveira, L.F.D. Moraes, V.L. Engel \& F.B. Gandara (orgs.). Restauração Ecológica de Ecossistemas Naturais. FEPAF, Botucatu, pp. 1-26.
Figueiredo Filho, A., Moraes, G.F., Schaaf, L.B. \& Figueiredo, D.J. 2003. Avaliação estacional da deposição de serapilheira em uma Floresta Ombrófila Mista localizada no sul do Estado do Paraná. Ciência Florestal 13: 11-18.

Frazer, G.W., Canhan, C.D. \& Lertzman, K.P. 1999. Gap light analyzer (GLA): Imaging software to extract canopy structure and gap light transmission indices from true color fisheye photographs, users manual and program documentation. Simon Fraser University, Burnaby, British Columbia and The Institute of Ecosystem Studies, Millbrook, New York.

Hobbs, R.J. 2008. The ecological context: a landscape perspective. In: M.R. Perrow \& A.J. Davy (eds.). Handbook of Ecological Restoration. Cambridge University Press, Cambridge, pp. 47-65.

IAPAR - Instituto Agronômico do Paraná. 2000. Cartas climáticas do estado do Paraná. http://www.iapar. $\mathrm{br} / \mathrm{modules} /$ conteudo/conteudo.php? conteudo $=677$. (acesso em 31.01.2013).

IBGE - Instituto Brasileiro de Geografia e Estatística. 2004. Mapa de vegetação do Brasil. http://www. ibge.gov.br/home/presidencia/noticias/21052004bio mashtml.shtm. (acesso em 31.01.2013).

IPCC - Intergovernmental Panel on Climate Change. 2007. The physical science basis. In: S. Solomon, D. Qin, M. Manning, Z. Chen, M. Marquis, K.B. Averyt, M. Tignor \& H.L. Miller (eds.). Contribution of working group I to the fourth assessment report of the intergovernmental panel on climate change. Cambridge University, Cambridge.

Jennings, S.B., Brown, N.D. \& Sheil, D. 1999. Assessing forest canopies and understory illumination: canopy closure, canopy cover and other measures. Forestry 72: $59-73$.

Kozlowski, T.T. \& Pallardy, S.G. 1996. Physiological of woody. 2ed. Academic, San Diego.

Kuruppuarachchi, K.A.J.M. \& Seneviratne, G. 2013. Predicting aboveground biomass increment of tropical forests from litter-fall. Ceylon Journal of Science 42: $35-40$.

Lv, G.H., Zhou, G.S. \& Wang, X.Y. 2013. Factors controlling litterfall production of forest in China. Advanced Materials Research 726: 4248-4251.

Machado, P.L.O.A. 2005. Carbono do solo e a mitigação da mudança climática global. Química Nova 28: 329-334.

Maciel, M.N.M., Watzlawick, L.F., Schoeninger, E.R. \& Yamaji, F.M. 2002. Efeito da radiação solar na dinâmica de uma floresta. Revista Ciências Exatas e Naturais 4: 102-114.

Martins, S.V. \& Rodrigues, R.R. 1999. Produção de serapilheira em clareiras. Revista Brasileira de Botânica 22: 405-412. 
Martins, S.V. 2012. Restauração ecológica de ecossistemas degradados. Editora UFV, Viçosa.

Melo, A.C.G. \& Durigan, G. 2006. Fixação de carbono em reflorestamentos de matas ciliares no Vale do Paranapanema, SP, Brasil. Scientia Forestalis 71: 149-154.

Melo, A.C.G. \& Durigan, G. 2007. Evolução estrutural de reflorestamentos de restauração de matas ciliares no Médio Vale do Paranapanema. Scientia Forestalis 73: 101-111.

Melo, A.C.G., Miranda, D.L.C. \& Durigan, G. 2007. Cobertura de copas como indicador de desenvolvimento estrutural de reflorestamentos de restauração de matas ciliares no médio vale do Paranapanema, SP, Brasil. Revista Árvore 31: 321-328.

Mielniczuk, J., Bayer, C., Vezzani, F., Lovato, T., Fernandes, F.F. \& Debarba, L. 2003. Manejo de solo e culturas e sua relação com estoques de carbono e nitrogênio do solo. In: N. Curi, J.J. Marques, L.R.G. Guilherme, J.M. Lima, A.S.S. Lopes \& V.H. Alvarez (eds.). Tópicos em ciência do solo. Sociedade Brasileira de Ciência do Solo, Viçosa, pp. 209-248.

Nave, L.E., Vance, E.D., Swanston, C.W., \& Curtis, P.S. 2010. Harvest impacts on soil carbon storage in temperate forests. Forest Ecology and Management 259: 857-866.

Nepstad, D.C., Tohver, I.M., Ray, D., Moutinho, P. \& Cardinot, G. 2007. Mortality of large trees and lianas following experimental drought in an Amazon forest. Ecology 88: 2259-2269.

Nutto, L., Watzlawick, L.F., Grammel, R. \& Fenner, P.T. 2002. O mercado internacional de CO2: Impacto das florestas naturais e plantações. In: C.R. Sanquetta, L.F. Watzlawick, R. Balbinot, M.A.B. Ziliotto \& F.S. Gomes (eds.). As Florestas e o Carbono. UFPR, Curitiba, pp. 89-108.

Palace, M., Keller, M., Asner, G.P., Silva, J.N.M. \& Passos, C. 2007. Necromass in undisturbed and logged forests in the Brazilian Amazon. Forest Ecology and Management 238: 309-318.

Palace, M., Keller, M. \& Silva, H. 2008. Necromass production: studies in undisturbed and logged Amazon Forests. Ecological Applications 18: 873-884.
Palace, M., Keller, M., Hurtt, G. \& Frolking, S. 2012. A review of above ground necromass in tropical forests. In: P. Sudarshana, M. Nageswara-Rao \& J.R. Soneji (eds.). Tropical Forests, Online Publisher, pp. 215-252.

Pawson, S.M., Brin, A., Brockerhoff, E.G., Lamb, D., Payn, T.W., Paquette, A. \& Parrotta, J.A. 2013. Plantation forests, climate change and biodiversity. Biodiversity and Conservation 22: 1203-1227.

Portela, R.C.Q. \& Santos, F.A.M. 2007. Produção de serapilheira em fragmentos florestais. Revista Brasileira de Botânica 30: 271-280.

Rangel, O.J.P., Silva, C.A., Guimarães, P.T.G., Melo, L.C.A. \& Oliveira Junior, A.C. 2008. Carbono orgânico e nitrogênio total do solo e suas relações com os espaçamentos de plantio de cafeeiro. Revista Brasileira de Ciência do Solo 32: 2051-2059.

Raven, P.H., Evert, R.F. \& Eichhorn, S.E. 2007. Fotossíntese, Luz e Vida. 7ed. In: P.H. Raven, R.F. Evert \& S.E. Eichhorn (orgs.). Biologia Vegetal. Editora Guanabara Koogan S. A., Rio de janeiro, pp. 126-151.

Rebetez, M., Renaud, V., Von Arx, G. \& Dobbertin, M. 2012. Impact of forest cover on increases in temperature under the canopy. General Assembly Conference Abstracts 14: 4443.

Santos, G.A. \& Camargo, F.A.O. 1999. Fundamentos da matéria orgânica do solo. Gênesis, Porto Alegre.

Trugilho, P.F., Silva, D.A., Frazão, F.J.L. \& Matos, J.L.M. 1990. Comparação de métodos de determinação da densidade básica em madeira. Acta Amazônica 20: 307-319.

Vital, B.R. 1984. Métodos de determinação da densidade da madeira. Boletim Técnico SIF 1: 1-21.

White, B.L.A., Nascimento, D.L.D., Dantas, T.V.P. \& Ribeiro, A.D.S. 2012. Dynamics of the production and decomposition of litterfall in a brazilian northeastern tropical forest (Serra de Itabaiana National Park, SE). Acta Scientiarum, Biological Sciences 35: 195-201. 
